\title{
Assessment of multi-target distinguishing using deconvolved conventional beamforming
}

\author{
Hualin Lan ${ }^{1,2,3, *}$, Xueqi Zhang ${ }^{1,2,3}$, Ruonan $\mathrm{Li}^{1,2,3}$, Suyu $\mathrm{Jin}^{1,2,3}$, and $N a \mathrm{Li}^{1,2,3}$ \\ ${ }^{1}$ Acoustic Science and Technology Laboratory, Harbin Engineering University, Harbin 150001, China \\ ${ }^{2}$ Key Laboratory of Marine Information Acquisition and Security (Harbin Engineering University), Ministry of Industry and \\ Information Technology, Harbin 150001, China \\ ${ }^{3}$ College of Underwater Acoustic Engineering, Harbin Engineering University, Harbin 150001, China
}

\begin{abstract}
Multi-target distinguishing based on beamforming is a popular topic in array signal processing. Conventional beamforming as a frequently used method is robust but constrained by the Rayleigh limit. Deconvolved conventional beamforming is a better choice since point scattering function could be derived by deconvolution based on Lucy-Richardson, with narrower beam width and lower sidelobe levels. Besides, the robustness of the conventional beamforming is maintained. In this paper, a new method of combined deconvolved conventional beamforming with Dolph-Chebyshev weights is proposed. The proposed method could overcome the deficit of deconvolved conventional beamforming on low mainlobe of weak target caused by iteration. Firstly, principles of the method are given including conventional beamforming, deconvolved conventional beamforming and the proposed algorithm combined deconvolved conventional beamforming with Dolph-Chebyshev weights. Then, performance of the proposed method for bi-target signals with the equivalent strength, in terms of the effect of signal frequency on distinguishing performance of two closed spaced targets coexisted is analysed. For weak target detection existed strong interference, the superiority of the proposed algorithm is analysed. Finally, proposed method is validated with sea trial data of two ship target noise recorded by a 48 -element array.
\end{abstract}

\section{Introduction}

The information of signal in the time domain and space domain is fully used for underwater target detection and bearing estimation based on hydrophone array. As a popular array signal processing method, the conventional beamforming (CBF) has the advantages of simplicity and robustness. However, it could not distinguish multitarget within the same beam limited by the Rayleigh limit. Detection performance of this algorithm for weak target is limited by sidelobes of strong interference [1].

To overcome the deficit of CBF, many high resolution algorithms have been proposed, represented by subspace algorithms. The MUSIC algorithm utilizes the orthogonality of the signal subspace and the noise subspace. ESPRIT takes advantage of the invariance of subspace rotation. These algorithms usually gain narrow mainlobe and low sidelobes. But they are sensitive to array mismatch and require high signal-to-noise ratio (SNR). What's more, the subspace algorithms require known the number of targets [2-4].

Deconvolved conventional beamforming $(\mathrm{dCv})$ based on conventional beamforming and deconvolution is a new algorithm, which combines the advantages of conventional beamforming algorithms and high resolution algorithms [5]. Point scattering function (PSF) could be derived by deconvolution based on beam output response and array beam pattern, with narrower beam width and lower sidelobe levels. As the same time, the robustness of the conventional beamforming is maintained.

To improve the performance of $\mathrm{CBF}$ in the field of weak target detection, the $\mathrm{CBF}$ has been modified with weighting processing, such as Dolph-Chebyshev weights (DCW) and null steering weights [6,7]. The feature of DCW is able to control sidelobe level. This method only decreases the influence of the sidelobes.

The signals received by hydrophone array always include some unexpected interference which is common in real environment. The detection performance of algorithms for weak targets buried in strong interference has become a hot topic. For $\mathrm{dCv}$, as the number of iterations increases, the weak target output response of $\mathrm{dCv}$ decreases and the performance of weak target detection become worse. This paper proposed a new method which combines $\mathrm{dCv}$ with DCW (DCW-dCv). To meet the requirements of algorithm application, the hydrophone array should be a ULA to apply the DolphChebyshev weights. Better detection performance for weak target is derived with benefit of maintaining the narrow beam width of $\mathrm{dCv}$ at the same time.

\section{Deconvolved conventional beamforming combined with Dolph- Chebyshev weights}

Corresponding author: lanhualin@hrbeu.edu.cn 
A horizontal uniform line array (ULA) with $N$ elements spaced at $d$ is considered, where $d$ is the half of signal wavelength with respect to frequency $f$ and noise received by each element is independent. The array consists of isotropic elements of the same size and sensitivity. The target signal and interference signal are both assumed to be in the far field plane waves within the same narrow band frequency centred in $f$. And signal travels in a straight line as the sound propagation speed is constant in the water. In addition, the target and interference are at the same depth as the horizontal ULA, i.e., all in the flat ocean bottom.

\subsection{Conventional Beamforming}

The principle of conventional beamforming could be concluded as follows. Beam output response is derived by summing the time delays compensation or phase differences compensation between the signals received by hydrophones of the array, where the time delays or phase differences of received signals are determined by the alignment of the array and the incident angle. The angle with respect to the maximum of the beam output is the incident angle. Beamforming is a linear process in terms of targets.

For one target, the beam output response can be given by

$$
B(\theta)=\frac{\sin \left[N \pi(d / \lambda)\left(\sin \theta-\sin \theta_{0}\right)\right]}{N \sin \left[\pi(d / \lambda)\left(\sin \theta-\sin \theta_{0}\right)\right]}
$$

where $B(\theta)$ is $\mathrm{CBF}$ beam output response, $\theta$ is scan angle, $\theta_{0}$ is the bearing of target, $\lambda$ is sound signal wavelength and

$$
\lambda=\frac{c}{f}
$$

For $M$ targets, the beam output response can be given by

$$
B(\theta)=\sum_{i=1}^{M} \frac{\sin \left[N \pi(d / \lambda)\left(\sin \theta-\sin \theta_{i}\right)\right]}{N \sin \left[\pi(d / \lambda)\left(\sin \theta-\sin \theta_{i}\right)\right]}
$$

where $B(\theta)$ is $\mathrm{CBF}$ beam output response, $\theta$ is scan angle, $\theta_{i}$ is the bearing of $i^{\text {th }}$ target. According to equation (3), the multi-target beam output response is the sum of the single-target beam output responses.

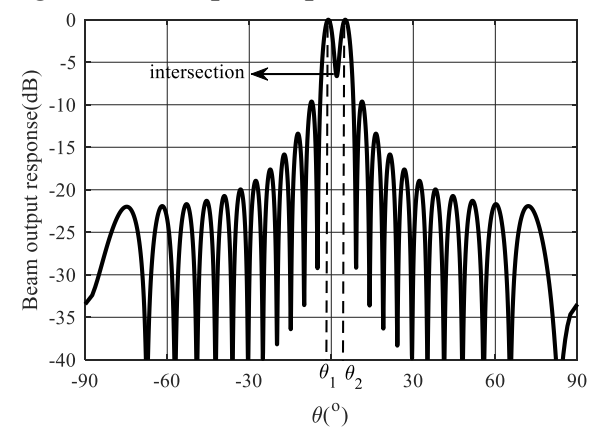

Fig. 1. Schematic diagram of $\mathrm{CBF}$ beam output response.

Two closed targets with equal strength are considered, the mainlobes of beam output response $B(\theta)$ of targets have an intersection as shown in Fig.1. The difference of $\theta_{1}$ and $\theta_{2}$ is defined as $\Delta \theta$. Normalize $B(\theta)$ and take the logarithm, if the value of intersection is smaller than $3 \mathrm{~dB}$, consider that these two closed targets could be distinguished. According to equation (3), the value of intersection could be seen as a function of $\theta_{i}$, affected by element number and signal frequency.For example, the number of elements is 24 , the space is $0.5 \mathrm{~m}$, the signal frequency is from $1000 \mathrm{~Hz}$ to $2000 \mathrm{~Hz}$, the bearing of one target is $0^{\circ}$, and another is varied. The value of intersection depending on $\Delta \theta$ under different signal frequency is shown in Fig.2. From this picture, the minimum $\Delta \theta$ which can distinguish two close targets is smaller as the increase of signal frequency.

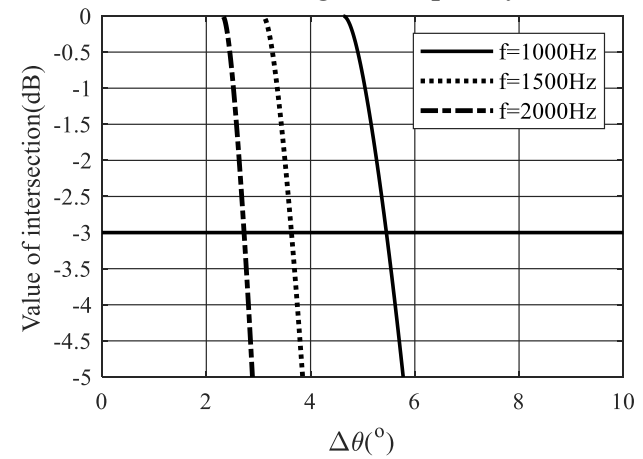

Fig. 2. Value of intersection as a function of $\Delta \theta$ under different signal frequencies.

\subsection{Deconvolved conventional beamforming}

As is well known, conventional beamforming results is the convolution of the beam pattern with PSF, the equation is given by

$$
B(\theta)=\int_{-\infty}^{\infty} R\left(\theta \mid \theta_{i}\right) \operatorname{PSF}\left(\theta_{i}\right) d \theta_{i}
$$

where $R(\theta)$ is array beam pattern and $\operatorname{PSF}(\theta)$ is PSF. And $\mathrm{dCv}$ makes the use of the known conventional beamforming results and array beam pattern to obtain the PSF by deconvolution.

In this paper, the Lucy-Richardson algorithm is used to achieve deconvolution [8]. This algorithm is an iterative algorithm given by

$$
\operatorname{PSF}^{(i+1)}(\theta)=P S F^{(i)}(\theta) \int_{-\infty}^{\infty} \frac{R\left(\theta \mid \theta_{i}\right)}{\int_{-\infty}^{\infty} R\left(\theta \mid \theta_{i}\right) P S F^{(i)}(\theta) d \theta_{i}} B(\theta) d \theta
$$

where $i$ is iteration number. With iteration number increasing, the recovered source information becomes more accurate. To make sure shift-invariant, the beam pattern is expressed in sine of $\theta$. The $\mathrm{dCv}$ result yields narrower mainlobe width and lower sidelobe levels.

\subsection{Deconvolved conventional beamforming combined with Dolph-Chebyshev weights}

For a ULA, one of the most commonly used weights is DCW [7]. Equal sidelobe levels can be obtained at the same time as required mainlobe-to-sidelobe ratio. This method makes use of the property of Chebyshev polynomial, which is defined as 


$$
T_{n}(x)= \begin{cases}\cos (n \arccos x) & |x| \leq 1 \\ \cosh (n \arccos h x) & |x|>1\end{cases}
$$

assumes that

$$
\begin{cases}x=\cos \varphi & |x| \leq 1 \\ x=\cosh \varphi & |x|>1\end{cases}
$$

then

$$
T_{n}(x)= \begin{cases}\cos (n \varphi) & |x| \leq 1 \\ \cosh (n \varphi) & |x|>1\end{cases}
$$

Chebyshev polynomial has recursive relationship as follows

$$
T_{n}(x)=2 x T_{n-1}(x)-T_{n-2}(x)
$$

and $T_{0}(x)=1$. When $|x| \leq 1$, it is easy to get

$$
\left\{\begin{array}{l}
T_{1}(x)=x=\cos \varphi \\
T_{2}(x)=2 x^{2}-1=2 \cos ^{2} \varphi-1 \\
\cdots
\end{array}\right.
$$

using the correspondence between Chebyshev polynomial and beam pattern, the DCW can be calculated as follows.

According to mainlobe-to-sidelobe ratio $A$, calculate $x_{0}$ by equation (11)

$$
x_{0} \approx \frac{1}{2}\left((2 A)^{\frac{1}{N}}+\left(\frac{1}{2 A}\right)^{\frac{1}{N}}\right)
$$

Calculate DCW $I_{N}^{n}$ by equation (12) and (13)

$$
\begin{gathered}
\alpha=1-\frac{1}{x_{0}^{2}} \\
I_{N}^{n}=\frac{N-1}{N-n} \sum_{s}\left(\begin{array}{l}
n-2 \\
s
\end{array}\right)\left(\begin{array}{l}
N-n \\
s+1
\end{array}\right) \alpha^{s+1} \frac{n !}{r !(n-r) !}
\end{gathered}
$$

where $1<n \leq N$ and $s=0,1,2, \ldots, N-1$.

The steps of DCW-dCv are as follows

a. set up mainlobe-to-sidelobe ratio, and calculate DCW according to equation (11), equation (12) and equation (13);

b. calculate array beam pattern based on DCW;

c. calculate beam output response based on DCW by beamforming;

d. calculate the DCW-dCv beam output response by using the array beam pattern and beam output response based on Lucy-Richardson algorithm.

\section{Simulation and results}

A horizontal ULA is considered in this section, the performance of multi-target distinguishing of $\mathrm{CBF}, \mathrm{dCv}$ and $\mathrm{DCW}-\mathrm{dCv}$ are studied as follows

- The dependence of minimum angular separation which can distinguish bi-target signals with equivalent strength on target signal frequency.

- The detection performance for weak targets detection of $\mathrm{dCv}$ and $\mathrm{DCW}-\mathrm{dCv}$ affected by SNR and number of iterations.

\subsection{Performance for bi-target distinguishing}

The minimum angular separation is defined as the standard of distinguishing performance for bi-target with equivalent strength, record as $\Delta \theta$. In this section, the number of elements is 24 , the space of elements is $0.5 \mathrm{~m}$, the signal frequency is from $500 \mathrm{~Hz}$ to $3000 \mathrm{~Hz}$, and the iteration number is 100 . The performance of $\mathrm{CBF}$ and $\mathrm{dCv}$ for bi-target with equivalent strength distinguishing is studied.

Comparison of $\mathrm{CBF}$ and $\mathrm{dCv}$ algorithm is given in Fig.3. Minimum angular separation as a function of the signal frequency is given in picture. The $\Delta \theta$ is reduced with the increase of signal frequency both of $\mathrm{CBF}$ and $\mathrm{dCv}$. After the deconvolution, the $\Delta \theta$ becomes smaller due to the narrower main beam. It is shown that the performance of $\mathrm{dCv}$ is superior to CBF. What's more, it is shown that the $\Delta \theta$ of $\mathrm{CBF}$ is consistent with theoretical value obtained from Fig.2.

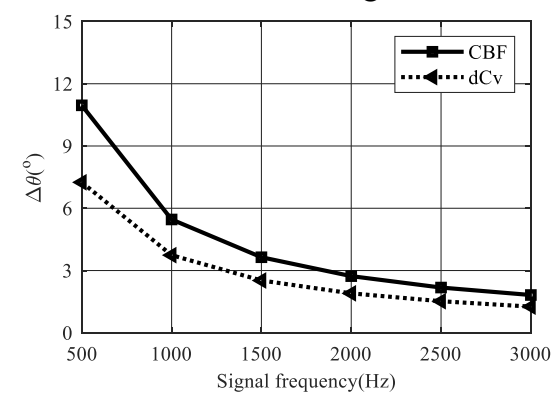

Fig. 3. $\Delta \theta$ as a function of signal frequency.

\subsection{Performance for weak targets detection}

The performance of $\mathrm{dCv}$ and $\mathrm{DCW}-\mathrm{dCv}$ for weak target detection is evaluated with RMSE and output response of weak target bearing, considered the influence of SNR and the number of iterations. The target signal arrives at DOA of $10^{\circ}$, the interference signal arrives at DOA of $0^{\circ}$. The signal-to-interference ratio (SIR) is $-20 \mathrm{~dB}$, and the mainlobe-to-sidelobe ratio is $-30 \mathrm{~dB}$.

\subsubsection{The influence of SNR on RMSE of weak target bearing}

In this section, the influence of SNR on RMSE of weak target bearing is studied. The number of elements is 24 , the space of elements is $0.5 \mathrm{~m}$, the target signal frequency and interference signal frequency are both $1500 \mathrm{~Hz}$, and the iteration number is 100 . The SNR is from $-10 \mathrm{~dB}$ to $10 \mathrm{~dB}$.

Comparison of $\mathrm{dCv}$ and DCW-dCv is given in Fig.4. The RMSE of weak target bearing depended on SNR is shown in the picture. The RMSE is reduced with the increase of SNR both for $\mathrm{dCv}$ and DCW-dCv. The RMSE of $\mathrm{dCv}$ tends to be constant with the value of $0.045^{\circ}$ when SNR is greater than OdB. RMSE of DCW$\mathrm{dCv}$ tends to be constant with the value of $0.077^{\circ}$ when SNR is greater than 0dB. What's more, it is also shown that the RMSE of DCW-dCv is smaller than $\mathrm{dCv}$ when SNR is higher than $-7 \mathrm{~dB}$. 


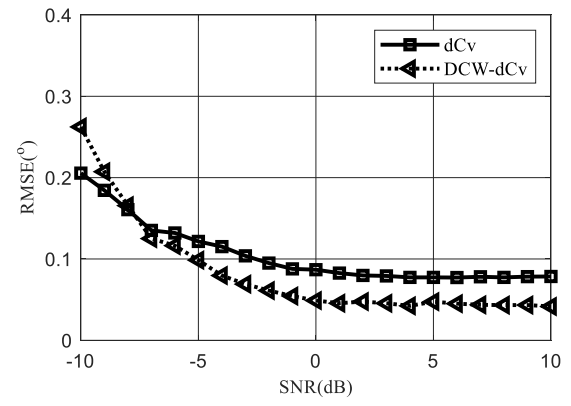

Fig. 4. RMSE as a function of SNR for weak target.

\subsubsection{The influence of number of iterations on RMSE of weak target bearing}

In this section, the influence of the number of iterations on RMSE of weak target output response is studied. The number of elements is 24 , the space of elements is $0.5 \mathrm{~m}$, the target signal frequency and interference signal frequency are both $1500 \mathrm{~Hz}$, and input SNR is $20 \mathrm{~dB}$. The number of iterations is from 20 to 200 .

Comparison of $\mathrm{dCv}$ and $\mathrm{DCW}-\mathrm{dCv}$ is given in Fig.5. RMSE of weak target bearing depended on number of iterations is shown in the picture. RMSE of $\mathrm{dCv}$ tends to be constant with the value of $0.6^{\circ}$ when the number of iterations is greater than 110 . RMSE of DCW-dCv tends to be constant with the value of $0.15^{\circ}$ when the number of iterations is greater than 40. It is shown that the RMSE of $\mathrm{dCv}$ for weak target is worse than $\mathrm{DCW}-\mathrm{dCv}$.

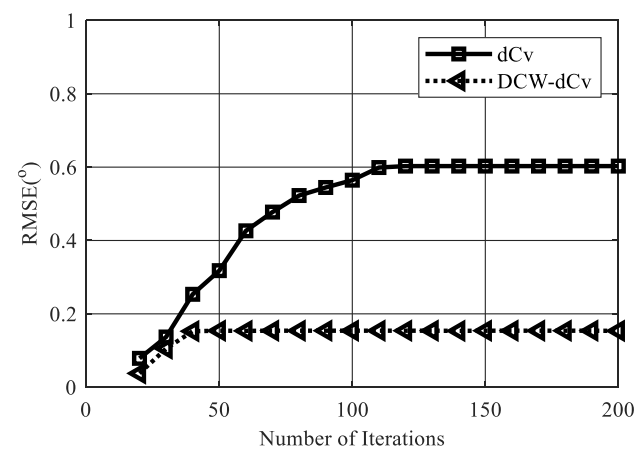

Fig. 5. RMSE as a function of number of iterations.

\subsubsection{The influence of number of iterations on weak target output response}

In this section, the influence of number of iterations on weak target output response is studied. The number of elements is 24 , the space of elements is $0.5 \mathrm{~m}$, the target signal frequency and interference signal frequency are both $1500 \mathrm{~Hz}$, and input SNR is $20 \mathrm{~dB}$. The number of iterations is from 20 to 200 .

Comparison of $\mathrm{dCv}$ and $\mathrm{DCW}-\mathrm{dCv}$ is given in Fig.6. Output response of weak target depended on the number of iterations is shown in the picture. The output response of $\mathrm{dCv}$ decreases from $-29 \mathrm{~dB}$ to $-38 \mathrm{~dB}$ as the number of iterations increases. After the $\mathrm{DCW}$, the weak target output response is a constant with the value of $-24 \mathrm{~dB}$. It is shown that the weak target detection of $\mathrm{DCW}-\mathrm{dCv}$ is better than $\mathrm{dCv}$.

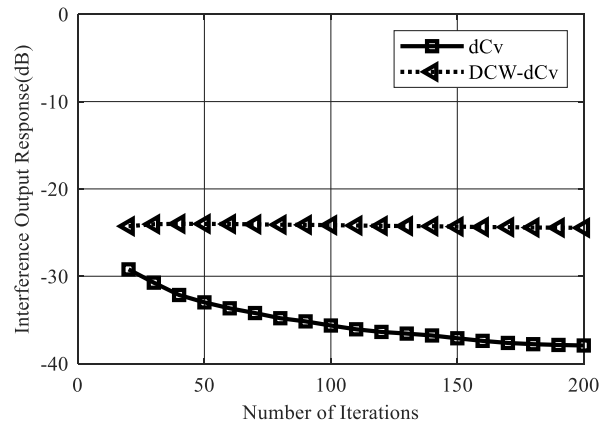

Fig. 6. Weak target output response as a function of number of iterations.

\section{Real data analysis}

In this section, the performance of the $\mathrm{CBF}, \mathrm{dCv}$ and $\mathrm{DCW}-\mathrm{dCv}$ is evaluated with real data. The data were collected on a horizontal ULA at sea. The array had 48 elements uniformly spaced at $0.25 \mathrm{~m}$. The signal propagation speed is $1500 \mathrm{~m} / \mathrm{s}$. The analysis frequency band of received signals is from $1 \mathrm{kHz}$ to $1.5 \mathrm{kHz}$.

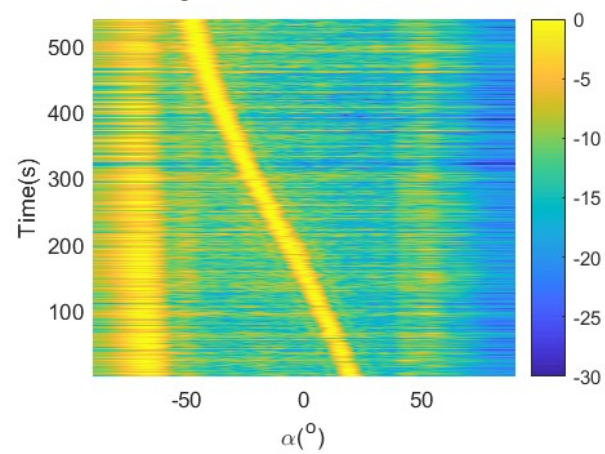

(a)

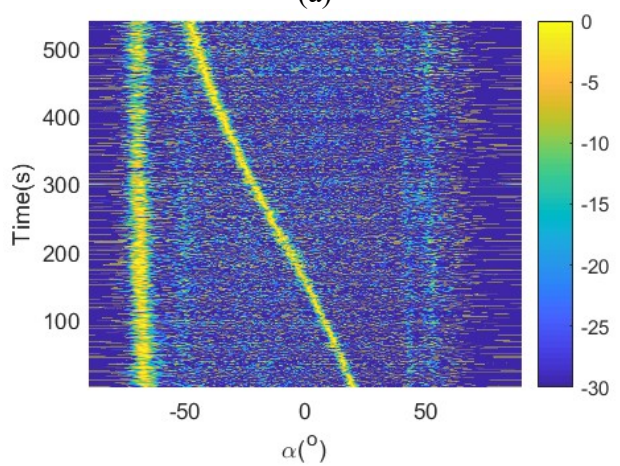

(b)

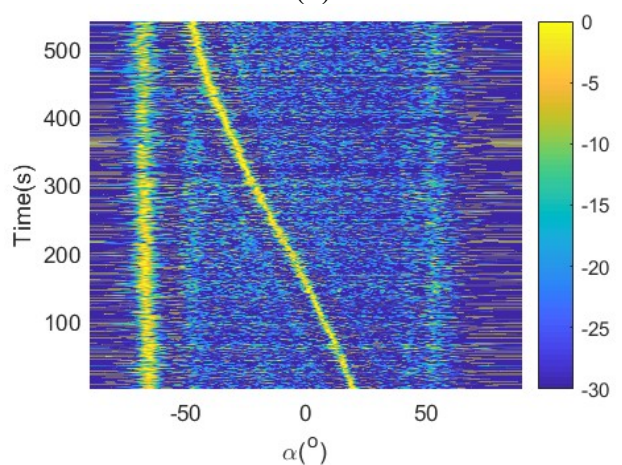

(c)

Fig. 7. BTR using (a) CBF, (b) dCv and (c) DCW-dCv. 
Comparison of $\mathrm{CBF}, \mathrm{dCv}$ and $\mathrm{DCW}-\mathrm{dCv}$ is given in Fig.7. Direction histories of two targets are shown in the picture. One is with nearly constant direction of $-67.5^{\circ}$ and another one is varied from $20^{\circ}$ to $-46.7^{\circ}$ during the observation time $0-540 \mathrm{~s}$. The estimated direction results of CBF in Fig.7(a) for the both two targets are much broader than other two methods and the background is much higher because of the effect of the sidelobe. After the deconvolution, the targets are much more distinct from the interference in Fig.7(b) and Fig.7(c). What's more, with the proposed method of the DCW-dCv, the mainlobe is narrower and more evident in Fig.7(c). It is shown that the data include two targets. Fig.7(a) shows the bearing time record (BTR) using CBF could estimate the bearing of moving target clearly, but another target could not be detected clearly. Fig.7(b) and Fig.7(c) show BTR using both $\mathrm{dCv}$ and $\mathrm{dCv}$ combined with $\mathrm{DCW}$ could distinguish two targets.

\section{Summary}

A new deconvolution beamforming method utilizing the Dolph-Chebyshev (DCW-dCv) was proposed in this paper to deal with the weaken of the beam output caused by iteration of $\mathrm{dCv}$ and its ability of multi-target distinguishing was assessed. Higher precision of DOA and more rapid convergence speed were achieved by the $\mathrm{DCW}-\mathrm{dCv}$ compared with $\mathrm{dCv}$ at the same signal-tonoise ratio. The superiority of $\mathrm{DCW}-\mathrm{dCv}$ is validated by simulation and sea trial data.

The work of this paper is supported by China NSFC (No.51609052).

\section{References}

1. M. S. Bartlett. Nature, 161 (1948):686-687

2. G. Liang, K. Zhang, Z. Fan, Journal of Harbin Engineering University, 33 (2012):30-36

3. G. Qiao, L. Zhang. Journal of Harbin Engineering University, 28 (2007):296-300.

4. H. Cox, R. M. Zeskind, IEEE Transactions on Acoustics Speech and Signal Processing, 36 (1988):10-19

5. T. C. Yang, IEEE Journal of Oceanic Engineering, PP (2017):1-13

6. R. Eirey-Perez, J. A. Rodriguez-Gonzalez, F. J. Ares-Pena, IEEE Transactions on Antennas and Propagation, 60 (2012):2106-2109

7. H. L. Van Trees. Optimum array processing, (Wiley, New York, 2002)

8. E. B. Richard. Theory of remote image formation, (Cambridge University Press, New York, 2004) 